\title{
Medium chain acyl-CoA dehydrogenase deficiency
}

INSERM

\section{Source}

INSERM. (1999). Orphanet: an online rare disease and orphan drug data base. Medium chain acyl-COA dehydrogenase deficiency. ORPHA:42

Medium chain acyl-CoA dehydrog enase (MCAD) deficiency (MCADD) is an inborn error of mitochondrial fatty acid oxidation characterized by a rapidly progressive metabolic crisis, often presenting as hypoketotic hypoglycemia, lethargy, vomiting, seizures and coma, which can be fatal in the absence of emergency medical intervention. 